\title{
Titanium isotope source relations and the extent of mixing in the proto-Solar nebula examined by Independent Component Analysis.
}

\author{
Robert C. J. Steele and Patrick Boehnke \\ Department of Earth, Planetary, and Space Sciences, University of California, Los Angeles, CA \\ 90095 \\ February 11, 2015
}

\begin{abstract}
The Ti isotope variations observed in hibonites represent some of the largest isotope anomalies observed in the Solar System. Titanium isotope compositions have previously been reported for a wide variety of different early Solar System materials, including calcium, aluminium rich inclusions (CAIs) and CM hibonite grains, some of the earliest materials to form in the Solar System, and bulk meteorites which formed later. These data have the potential to allow mixing of material to be traced between many different regions of the early Solar System. We have used Independent Component Analysis to examine the mixing end-members required to produce the compositions observed in the different datasets. The ICA yields results identical to a linear regression for the bulk meteorites. The components identified for hibonite suggest that most of the grains are consistent with binary mixing from one of three highly anomalous nucleosynthetic sources. Comparison of these end-members show that the sources which dominate the variation of compositions in the meteorite parent body forming regions was not present in the region in which the hibonites formed. This suggests that the source which dominates variation in $\mathrm{Ti}$ isotope anomalies between the bulk meteorites was not present when the hibonite grains were forming. One explanation is that the bulk meteorite source may not be a primary nucleosynthetic source but was created by mixing two or more of the hibonite sources. Alternatively, the hibonite sources may have been diluted during subsequent nebula processing and are not a dominant Solar System signatures.
\end{abstract}

Subject headings: astrochemistry; meteorites, meteors, meteoroids; methods: statistical; nuclear reactions, nucleosynthesis, abundances; protoplanetary disks; supernovae: general

\section{Introduction}

Isotope anomalies in primitive meteorites and their components can be used to examine the nucleosynthetic origins of the Solar System (e.g. Lee et al. 1978). The various materials we sample in meteorites, for example CAIs, chondrules and achondrites, formed in different parts of the proto-Solar nebula, and at different times. Therefore, these samples naturally record some of the variation in isotopic compositions present in the early Solar System. Investigation of the differences in anomalies between these distinct materials can be used to examine their genetic relationships and the processes by which the different 
sources were mixed together to yield the variation in compositions we observe today.

Isotope anomalies in refractory elements with more than four isotopes, for example $\mathrm{Ti}$, $\mathrm{Ni}$ and Mo, are highly correlated through the bulk meteorites, both chondrites and achondrites, and the population of normal CAIs (e.g. Trinquier et al. 2009; Steele et al. 2010, 2011, 2012; Burkhardt et al. 2011). This systematic variation occurs in most early Solar System objects. There are, however, a few notable exceptions to this systematic variation, most interestingly the FUN (fractionated with unknown nuclear effects) CAIs and the CM hibonite grains. These are relatively poorly understood populations of refractory inclusions found in primitive meteorites.

Hibonite, the focus of this study, is a highly refractory minerals thought to have been one of the first minerals to have formed in the Solar System either as a refractory residue or by condensation from a nebula gas (Ireland et al. 1988). Hibonite grains from the CM carbonaceous chondrites (CCs) exhibit some of the largest isotope anomalies of any materials thought to have formed within the Solar System. The evidence that the hibonite grains are of Solar origin stems from their $\mathrm{O}$ isotope compositions within the normal range for Solar System objects (Fahey et al. 1987a; Ireland et al. 1992; Liu et al. 2009). However, they exhibit ${ }^{50} \mathrm{Ti}$ enrichments of up to $27 \%$ (Ireland 1990) and deficits of up to 7 \% (Hinton et al. 1987). Several populations of hibonite grains have been documented in the literature (Ireland et al. 1988; Marhas et al. 2002), though they can broadly be split into two groups based on their petrology and their isotopic compositions. The first group is petrologically characterised by platy hibonite crystal fragments (PLACs from Ireland et al. 1988) and blue aggregates (BAGs from Ireland et al. 1988). This group shows the large mass-independent isotope anomalies in elements such as $\mathrm{Ti}$ and $\mathrm{Ca}$ described above and no evidence for live short-lived radionuclides (SLRs) such ${ }^{26} \mathrm{Al}$ (Ireland et al. 1988; Ireland 1990). The second group, comprised of spinel and hibonite rich spherules (SHIBs from Ireland et al. 1988), show much smaller massindependent isotope anomalies but do show evidence for live SLRs for example canonical, or supra-canonical ${ }^{26} \mathrm{Al}$ (Ireland et al. 1988; Ireland 1990; Liu et al. 2009).

The large variation of mass-independent isotope anomalies found in hibonites are thought to represent either pure, or only slightly diluted, presolar signatures (e.g. Ireland 1990). However, it is not known if the events which produced the hibonites occurred within the Solar System at some time before the large scale chemical and isotopic homogenisation. Alternatively, the hibonites may have formed outside the Solar System, possibly closer to the source of the isotopic anomalies, for example around a star in some region of the molecular cloud which had similar oxygen isotope compositions. Therefore, it is clearly of great importance to assess the relationship of the anomalies observed in the hibonites to those observed in bulk meteorites and other Solar System materials. Examining these relationships will offer insight into whether the hibonites represent purer samples of the isotopic precursors of the bulk Solar System and sampled by the bulk meteorites or if they represent a previously unsampled reservoir. An example of this type of finding is the recent discovery of oxide grains highly enriched in ${ }^{54} \mathrm{Cr}$ which are thought to be the carrier phase of mass-independent $\mathrm{Cr}$ isotope anomalies in the Solar System (Dauphas et al. 2010; Qin et al. 2011). The alternative, that the hibonites formed outside the Solar System, possibly prior to Solar System formation, would mean that hibonite grains do not have isotopic significance for the bulk Solar System. Rather they represent a new population of presolar grain that is physically an order of magnitude larger than the previous largest population (with exception of certain very rare 
examples e.g., Zinner et al. 2010). However, previous presolar grain populations have very large $\mathrm{O}$ isotope variations, several orders of magnitude larger than those observed in CM hibonites (Fahey et al. 1987b; Zinner 2003)

In order to examine the relationships between hibonite grains and bulk meteorites common data must be examined to find similarities. With the exception of $\mathrm{O}, \mathrm{Ti}$ is the element which has been most extensively studied in a wide variety of early Solar System materials, including hibonite, grains FUN and normal CAIs and bulk meteorites. Though O has been more extensively studied, it is not suitable for this purpose for two reasons. Firstly, the origins of $\mathrm{O}$ isotope variations are debated and may have non-unique sources (Thiemens 1999; Lyons and Young 2005; Lyons et al. 2009). Moreover, there has clearly been subsequent mixing between these different Solar System reservoirs. Secondly, O only has three isotopes, so only one mass-independent ratio, which limits the scope for analysing sources. For these reasons we focus on $\mathrm{Ti}$.

The first studies of $\mathrm{Ti}$ were in the FUN CAIs by the ANU and Caltech groups (Heydegger et al. 1979; Niemeyer and Lugmair 1980). These rare inclusions exhibited large anomalies in $\mathrm{Ti}, \pm 40 \%$ on ${ }^{50} \mathrm{Ti}$, where $\%$ oo is the $\varepsilon$ unit defined as the parts per ten thousand difference to a terrestrial standard. Several other elements show similar variation, for example $150 \% 00$ on ${ }^{48} \mathrm{Ca}$ (Lee et al. 1978) and $290 \%$ on ${ }^{58} \mathrm{Fe}$ (Völkening and Papanastassiou 1989). Interestingly, these large anomalies on ${ }^{58} \mathrm{Fe}$ are unique in the Solar System as no other objects have been found to show iron isotopic variation (e.g. Dauphas et al. 2008). Even larger anomalies were found in the hibonite grains from CM chondrites (e.g. Fahey et al. 1985), see above. Smaller, but still significant, Ti isotope anomalies were subsequently observed in the normal population of CAIs (e.g. Niemeyer and Lugmair 1981) and in bulk meteorites (e.g. Leya et al. 2008).
In general, the early studies of $\mathrm{Ti}$ isotopes in chondritic inclusions and hibonites grains showed large anomalies on the neutron rich isotope ${ }^{50} \mathrm{Ti}$ and smaller anomalies on the other isotopes. However, no coherent correlations have been observed in the $\mathrm{Ti}$ isotope compositions from these samples. These studies were focused on examining the input of neutron-rich isotopes to the early Solar System, principally because of the early identification of anomalies on ${ }^{48} \mathrm{Ca}$ in FUN CAIs (Lee et al. 1978), which led to the almost universal use of normalization to ${ }^{46} \mathrm{Ti} /{ }^{48} \mathrm{Ti}$ for the massdependent fraction correction in early studies. Fahey et al. (1987a) examined the number of sources required to produce the variation in $\mathrm{Ti}$ isotope compositions by fitting the data to a plane with a least squares algorithm similar to the more conventional York regression for 2-d correlation. Fahey et al. (1987a) concluded that at least 4 , possibly more, sources were needed to reproduce the observed compositions.

In contrast, the more recent, high precision data for bulk meteorites show a striking correlation between $\varepsilon^{50} \mathrm{Ti}_{\frac{47}{49}}$ and $\varepsilon^{46} \mathrm{Ti}_{\frac{47}{49}}$ where the subscripts denote the normalizing ratio, see section 2.1 below (Leya et al. 2008, 2009; Trinquier et al. 2009; Zhang et al. 2011, 2012). This correlation was shown by Qin et al. (2011) and Steele et al. (2012) to be consistent with input from the $\mathrm{O} / \mathrm{Ne}$ zone of a type II supernova (SN II). Mass-independent isotopic variation between different bulk meteorite groups likely represents much more anomalous $\mathrm{Ti}$ by mass than the much larger anomalies seen in the hibonite grains.

One obvious hypothesis for the origin of $\mathrm{Ti}$ isotope variation in the Solar System is that one of the sources present in the hibonite grains is also the source represented by the variation observed in the bulk meteorites. Relationships between hibonites and other meteorite groups were investigated by previous studies, for example Fahey et al. (1987a). 
Since these early investigations more higher quality data have been reported, especially in the bulk meteorite populations, therefore this subject is worth revisiting. The aim of this paper is to test the hypothesis that one of the sources present in the compositions of the hibonites and FUN CAIs is the same source observed in the bulk meteorites. This will enable us to examine the reasons why multiple sources are observed in the meteoritic inclusions (hibonites and FUN CAIs) while only one appears to be sampled by the bulk meteorites.

\section{Methods}

\subsection{Renormalization}

Mass-independent isotope anomalies require normalisation for mass-dependent fractionation. This is normally achieved using one isotope ratio to calculate and remove the expected fractionation on the other ratios, a process termed 'internally normalising'. See Russell et al. (1978), McCulloch and Wasserburg (1978) and Young et al. (2002) for discussions of fractionation corrections and the choice of fractionation 'laws'. Many of the hibonite data were original presented normalized to ${ }^{46} \mathrm{Ti} /{ }^{48} \mathrm{Ti}$ by the exponential law. These data have been renormalized to ${ }^{47} \mathrm{Ti} /{ }^{49} \mathrm{Ti}$ to make them comparable with the more recent bulk data for which $\varepsilon^{50} \mathrm{Ti}_{\frac{47}{49}}$ and $\varepsilon^{46} \mathrm{Ti}_{\frac{47}{49}}$ anomalies have been observed. It is important for the Independent Component Analysis (ICA) (see below) that appropriate uncertainties propagated through the normalization. To ensure renormalized uncertainties are appropriate they have been modelled using a Monte Carlo simulation by varying the data around their original uncertainties and propagating this through the full renormalization. This was repeated 10000 times to fully map the parameter space and the renormalized uncertainty is then the standard deviation (s.d.) of these repeats. The code used for the renormalization was written using $\mathrm{R}$ ( $\mathrm{R}$ Core Team
2013) and is available on request. The renormalized data are given an appendix table and are plotted in figure 1 .

\subsection{Independent Component Analysis}

Independent Component Analysis is a method of blind source separation by which a multivariate dataset can be deconvolved into independent subcomponents (Comon 1994). That is, a dataset, in this case $\mathrm{Ti}$ isotope data from hibonites, can be examined for the sources which mixed to produce the observed variation. Independent Component Analysis is related to the more commonly used principal component analysis (PCA) but differs in that the components identified do not have to be orthogonal, thus making it a more flexible tool. Independent Component Analysis relies on two assumptions, firstly that the sources are statistically independent from each other, and secondly that mixing between sources is linear. Independent Component Analysis algorithms work by finding solutions which minimise the shared information between components and maximising the non-Gaussianity of the individual components. The ICA algorithm returns a component, or vector, along which a significant proportion of the variation observed in the dataset as a whole can be described. Where significant variation remains, more components can be added until the dataset can be fully described. The components describe a slope which is a mixing line to a composition which represents one of the sources required to produce the variation in the data. These vectors, or components, may share a source at one end, or all sources may be independent, so there must be at least $n+1$ sources where $n$ is the number of components. Therefore, three vectors may represent between 4 and 6 isotopic source compositions. To describe the vector, the ICA reports a point and the vector is the slope to the origin in $n$ dimensions. These points maybe in either positive or negative fields because the ICA only determines the vector and the sign is assigned 

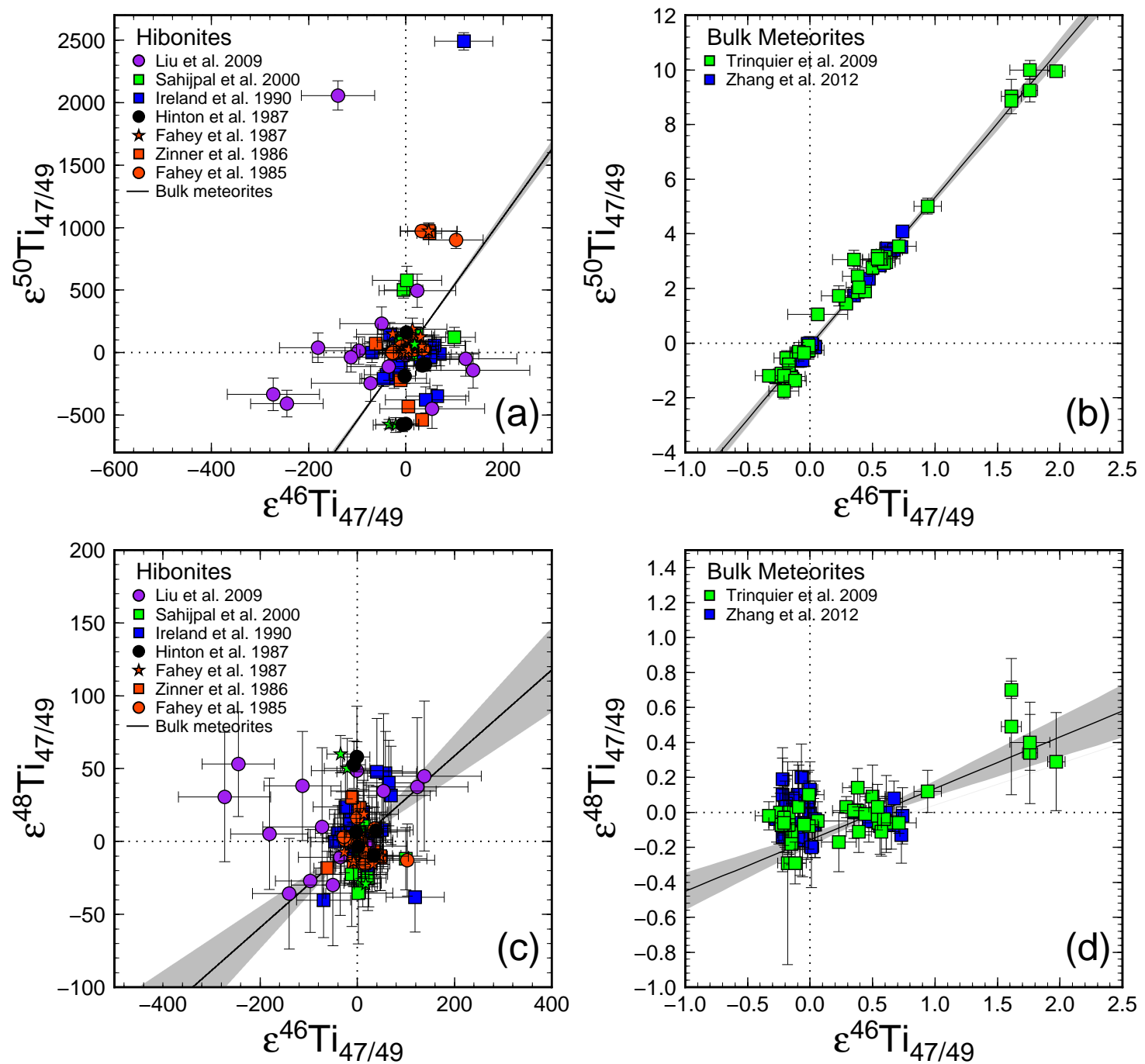

Fig. 1. - Figures showing previous Ti isotope compositions for hibonites (a) and (c) (from Fahey et al. 1985; Zinner et al. 1986; Fahey et al. 1987a; Hinton et al. 1987; Ireland 1990; Sahijpal et al. 2000; Liu et al. 2009), bulk meteorites and normal CAIs (b) and (d) (from Trinquier et al. 2009; Zhang et al. 2011, 2012). Also shown on both pairs of plots are extrapolations, with error envelopes, of the slopes from bulk meteorites and normal CAIs determined by York Regression (York 1969; Mahon 1996).

randomly. For a full description of ICA see Hyvärinen and Oja (2000).

There have been two previous studies in geochemistry and cosmochemistry which have used ICA for investigating source relationships. These looked at the distribution of mantle sources from the isotopic compositions of MORBs and OIBs (Iwamori and Albarède 2008) and the mixing relations in the HED suite of meteorites by examining major element abundances (Usui and Iwamori 2013). It is not clear that the requirement for straight line mixing is satisfied in the study of Iwamori and Albarède (2008) as they include isotope data from several different elements. Where different elements are used mixing will only approximate straight line mixing if the denominator isotopes are of the same concentration. 
It is important to note that in this study mixing is linear in Ti isotope space as all the ratios use the same denominator isotope.

We have used the FastICA algorithm of Hyvarinen (1999); Hyvärinen and Oja (2000) implemented in an R (R Core Team 2013) script by Marchini et al. (2013) to determine the independent components of Ti isotope data. A limitation of the traditional ICA is that uncertainties are not taken into account. This is because for the situations in which it has normally been used, the noise on the data does not come from measurement uncertainties, rather from real variation in the data.

We have extended the of traditional ICA by combining it with a Monte Carlo simulation of the uncertainties, or bootstrap (Efron 1981), of the data in order to gain a statistical measure of the uncertainty of the independent components determined by the FastICA algorithm. The isotopic data were varied randomly around their averages with a Gaussian distribution and an ICA performed for each iteration, this was repeated 20000 times to achieve a robust measure of the uncertainty. We have used clustering algorithms to sort the data into the individual components (Fritz et al. 2012; Cuesta-Albertos et al. 1997). During this process $1 \%$ of the models were discarded (e.g. tclust $\alpha=0.01$ ) because they did not fit into any of the clusters. This loss of data is taken into account in the eventual uncertainty. The clustering algorithms were optimised to use equal weights to ensure that the clusters contained equal numbers of points. The $\mathrm{R}$ scripts used to perform the ICA are available on request.

The results of these iterations produce a number of vectors which describe a mixing line towards the source and are illustrated by the distribution of points in figure 2.The vectors form clusters, the number of which is twice the number of components used because the vector may be either positive or negative from the origin; each pair of clusters describes the distribution of one component. The variation within a cluster of vectors describes the uncertainty in the component and includes contributions from the analytical uncertainty of the data and the uncertainty inherent in the ICA algorithm. This gives a robust measure of the uncertainty of the components determined by the ICA and allows components from different sample populations to be compared.

\subsection{Data used for the ICA}

We have performed the ICA on both the hibonite and bulk meteorite datasets. We have used hibonite data for SHIBs, PLACs and BAGs from as many studies possible including measurements from: Fahey et al. (1985); Zinner et al. (1986); Fahey et al. (1987a); Hinton et al. (1987); Ireland (1990); Sahijpal et al. (2000); Liu et al. (2009). The data from these studies were collected over 25 years on a variety of of ion probes, however, they all provide measurements from the standard Madagascar hibonite which are in agreement warranting their inclusion in this metadataset.

Several recent studies have measured the $\mathrm{Ti}$ isotope compositions of bulk meteorites and normal CAIs (Leya et al. 2008, 2009; Trinquier et al. 2009; Zhang et al. 2011, 2012). However, not all of these studies are appropriate to include as a metadataset. The recent $\mathrm{Ti}$ studies of bulk meteorites and normal CAIs all show broadly consistent results finding correlated anomalies on $\varepsilon^{50} \mathrm{Ti}_{\frac{47}{49}}$ and $\varepsilon^{46} \mathrm{Ti} \frac{47}{49}$, with very little variation on $\varepsilon^{48} \mathrm{Ti}_{\frac{47}{49}}$. Trinquier et al. (2009); Zhang et al. (2011, 2012) report very high precision $\mathrm{Ti}$ isotope compositions for a wide range of bulk meteorites, both chondrites and achondrites. In addition Trinquier et al. (2009) report Ti isotope compositions for the normal population of CAIs. Though the earlier studies of Leya et al. $(2008,2009)$ show broadly similar results to the more recent studies, they are of lower precision and so would hamper our ability to determine the slopes of mixing. Leya 
et al. (2008) also raise concerns over large non-terrestrial blanks introduced to some of their samples during digestion in Teflon bombs which may affect the accuracy of some data. Therefore we do not include the results of Leya et al. $(2008,2009)$ in our meta-dataset. Correlations produced by the studies of Trinquier et al. (2009); Zhang et al. $(2011,2012)$ are very consistent. In $\varepsilon^{46} \mathrm{Ti} \frac{47}{49} \mathrm{Vs} . \quad \varepsilon^{50} \mathrm{Ti} \frac{47}{49}$ they produce slopes of $5.48 \pm 0.27$ and $5.23 \pm 0.22$ for Trinquier et al. (2009) and Zhang et al. (2011, 2012) respectively. A "new" York regression (Mahon 1996) based on this combined dataset yields $5.37 \pm 0.15$.

\subsection{ICA Data Presentation and Mix- ing angles}

In order to compare the sources of different sample populations, the mixing vectors of each cluster, and the variation in these vectors within each cluster, must be compared. The data are presented initially by simply plotting the points returned by the ICA. In order that the components obtained from different sample populations maybe compared on the same diagram, the data for each point, in each individual dimension, has been normalized to the sum of the standard deviations of each dimension. This was achieved using

$$
\gamma\left({ }^{j} \operatorname{Ti} \frac{47}{49}\right)=\frac{i\left({ }^{j} \operatorname{Ti} \frac{47}{49}\right)}{\sigma\left({ }^{j} \operatorname{Ti}_{49}^{47}\right)+\sigma\left({ }^{k} \operatorname{Ti}_{49}^{47}\right)+\sigma\left({ }^{l} \operatorname{Ti}_{49}^{47}\right)},
$$

where $\gamma\left({ }^{j} \mathrm{Ti} \frac{47}{49}\right)$ is the renormalized component composition for the $i$ th iteration of the ICA in the dimension of $\left({ }^{j} \mathrm{Ti}_{49} \frac{47}{49}\right)$ (where isotope ${ }^{j} \mathrm{Ti}$ which was normalized for mass-dependent fractionation using the ${ }^{47} \mathrm{Ti} /{ }^{49} \mathrm{Ti}$ ratio), $j, k$ and $l$ represent the isotopes of $\mathrm{Ti}, i\left({ }^{j} \mathrm{Ti}_{\frac{47}{49}}\right)$ is the raw composition of the $i$ th iteration of the ICA in the dimension of $\left({ }^{j} \mathrm{Ti}_{\frac{47}{49}}\right)$ and $\sigma\left({ }^{j} \mathrm{Ti}_{\frac{47}{49}}\right)$, $\sigma\left({ }^{k} \mathrm{Ti}_{49} \frac{47}{49}\right)$ and $\sigma\left({ }^{l} \mathrm{Ti} \frac{47}{49}\right)$ is the standard deviation over all iterations of the ICA in the dimensions of $\left({ }^{j} \mathrm{Ti}_{49}^{47}\right),\left({ }^{k} \mathrm{Ti}_{\frac{47}{49}}\right)$ and $\left({ }^{l} \mathrm{Ti}_{\frac{47}{49}}\right)$, respectively. Thus the total variation of each sample population can be presented in the same, albeit artificial range. Importantly though, the angular information is retained, so the slopes, or angles, of the components hold through this normalization.

In order to quantitatively compare the components obtained for different sample populations the vectors must be obtained from the slopes. Previous studies have used the approach of comparing slopes of mixing in order to compare data sets (e.g. Dauphas et al. 2004; Steele et al. 2012). In these previous cases the dataset being compared were bulk meteorite data and modelled nucleosynthetic data for different astrophysical environments, not different populations of measurements but the concepts are the same. The use of slopes has some associated problems for comparison of different datasets. The major problem with slopes is that when they approach the vertical or horizontal the slope loses linearity and tends to zero or infinity. This reduces the ability to graphically resolve the difference between a slopes multiple slopes of different magnitudes. More seriously, however, if the uncertainty of a slope crosses either the vertical axis it will artificially encompass both zero and infinity making statistical comparison between different more complicated.

The issue of infinities may be simplified by using the angles of mixing (where $\theta=\arctan (\mathrm{m}) \cdot 180 / \pi)$ rather than slopes of mixing $(\mathrm{m})$. Angles of mixing have the advantage that they are linear and contain only one singularity (360 to 0 , where 0 is the positive limb of the $\mathrm{x}$ axis) compared to the four of slopes. This means that for comparison of two mixing lines (and possibly more) a position of the singularity may be chosen such none of the uncertainties are affected. In this study mixing lines are presented as angles of mixing and not slopes. This method could be useful in other areas, for example the comparison of mixing of nucleosynthetic environments in to the Solar System. 


\section{Results of the ICA}

The results of the ICA are presented in table 1 and figures 2 (a), (b), (c), (d) and 3 .

\begin{tabular}{lrrr}
\hline \hline & Average & -2 s.d. & +2 s.d. \\
\hline$\varepsilon^{50} \mathrm{Ti} \frac{47}{49} / \varepsilon^{46} \mathrm{Ti} \frac{47}{49}$ & & & \\
Bulk $\{\mathrm{a}\}$ & 79.784 & 0.323 & 0.347 \\
Bulk $\{\mathrm{b}\}$ & 79.467 & 0.519 & 0.442 \\
Hibonite $\{1\}$ & 88.673 & 1.126 & 0.962 \\
Hibonite $\{2\}$ & 24.342 & 56.666 & 31.094 \\
Hibonite $\{3\}$ & 101.968 & 18.662 & 26.189 \\
\hline$\varepsilon^{50} \mathrm{Ti} \frac{47}{49} / \varepsilon^{48} \mathrm{Ti} \frac{47}{49}$ & & & \\
Bulk $\{\mathrm{a}\}$ & 86.069 & 2.059 & 1.146 \\
Bulk $\{\mathrm{b}\}$ & 92.264 & 1.492 & 4.145 \\
Hibonite $\{1\}$ & 91.040 & 0.459 & 0.463 \\
Hibonite $\{2\}$ & 100.075 & 60.164 & 43.414 \\
Hibonite $\{3\}$ & 106.891 & 6.463 & 18.400 \\
\hline
\end{tabular}

Table 1: Results of the ICA on the hibonite and bulk meteorite populations. The results are presented as the angle $\left(\theta^{\circ}=\arctan (\mathrm{m}) \cdot 180 / \pi\right.$, where $\mathrm{m}$ is the slope) of mixing vectors to the returned components. The uncertainties are $95 \%$ confidence intervals derived from the spread of the ICA components returned by the bootstrap. Note the errors are the deviation in angle and are not symmetrical.

\subsection{ICA components}

Figures 2 (a), (b) and (c) show the ICA components presented as $\gamma\left({ }^{j} \mathrm{Ti}_{\frac{47}{49}}\right)$ normalized data show the hibonite and bulk meteorite data.

As expected the results of the ICA for the bulk meteorite data, shown in red colours in figure 2(a), show a very clear correlation between $\gamma\left({ }^{50} \mathrm{Ti}_{49} 47\right)$ and $\gamma\left({ }^{46} \mathrm{Ti}_{49} \frac{47}{49}\right)$. In the figures 2 (b) and (c), which include the other dimension $\gamma\left({ }^{48} \mathrm{Ti}_{\frac{14}{49}}\right)$, the components are not correlated. The divergence from a single correlation, and the presence of well defined components, shows that variation in $\varepsilon^{48} \mathrm{Ti}_{49}^{47}$ is observed and the mixing vectors of the sources can be examined by the ICA.

\subsection{ICA angles}

The single, tight, correlation observed between $\varepsilon^{46} \mathrm{Ti}_{\frac{47}{49}}$ and $\varepsilon^{50} \mathrm{Ti}_{\frac{47}{49}}$ in the bulk meteorites can be used as a test of the accuracy of the ICA method for determining the mixing slopes of the sources and also the uncertainty estimation by our bootstrap method, see figure 3. Regardless of any variation in $\varepsilon^{48} \mathrm{Ti}_{49}$, a projection of the plane present in the bulk meteorites on to the $\varepsilon^{50} \mathrm{Ti}_{\frac{47}{49}}$ vs. $\varepsilon^{46} \mathrm{Ti}_{\frac{47}{49}}$ axis will be a single correlation. This holds for line fitting algorithms, such as the least squares York regression, and ICA. Therefore, the slopes and errors obtained by York regression and ICA can be compared. This offers a demonstration of the accuracy of the angle obtained by the ICA and the precision of this method relative to the uncertainty of the original data. A "new" York regression on the combined dataset of Trinquier et al. (2009) and Zhang et al. (2012) yields a slope of $5.37 \pm 0.15$, the angle obtained from this slope is $79.45 \pm 0.29^{\circ}$. This value is vey close to the average value obtained by the ICA of $79.63 \pm 0.42^{\circ}$, see figure 3. Moreover, the uncertainties are also very similar, with the uncertainty slightly larger as expected as it includes a potential contribution from the ICA algorithm. This displays the ability of ICA to return accurate and precise vectors of source mixing.

\section{Discussion}

The results of the ICA show clear differences between character of the sources represented in the hibonite population versus the bulk meteorites and the normal CAIs, see figure 2. The most obvious explanation for this would be that the sources represented by the hibonite and bulk meteorites populations are different. The implications of this explanation are discussed in detail in section 4.3. However, there are several other possible reasons why the components represented by these two populations might be different. Firstly, the sources represented by the bulk meteorites and 

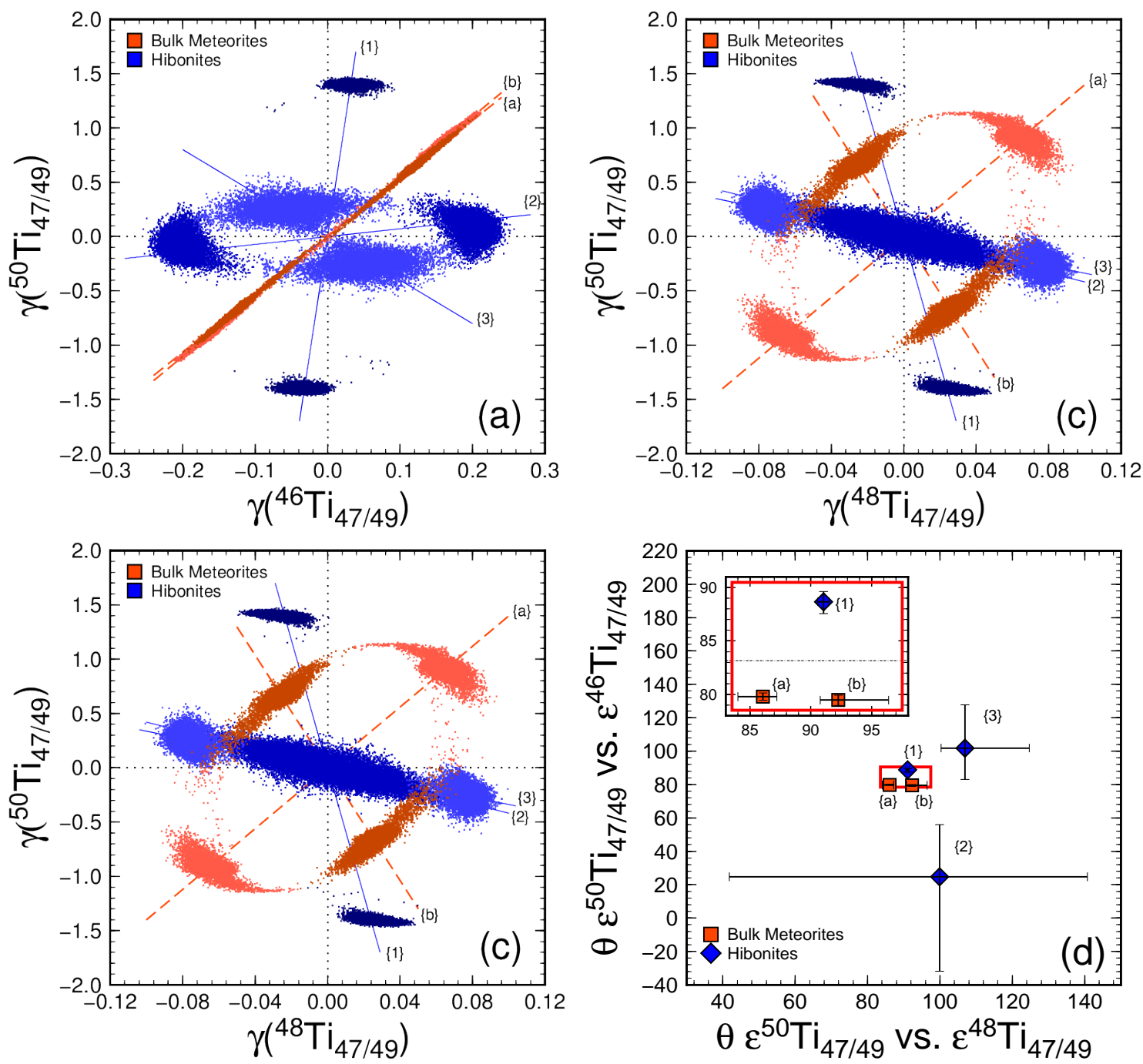

Fig. 2.- Figures showing the results of the ICA bootstrap for both the hibonite and bulk meteorite data. Plots (a), (b) and (c) show all the raw compositions of the ICA bootstrap iterations with the exception, in the case of the hibonites, of $1 \%$ of components which are so far away from an average component composition that they cannot be grouped. The individual components are highlitghted by diffrent shades and labelled with $\{1\},\{2\},\{3\},\{a\}$ and $\{b\}$. Plot (d) shows the slopes of mixing of the different components and their uncertainties. By plotting $\theta \varepsilon^{50} \mathrm{Ti} \frac{47}{49} \mathrm{vs}$. $\varepsilon^{46} \mathrm{Ti} \frac{47}{49}$ against $\theta \varepsilon^{50} \mathrm{Ti} \frac{47}{49}$ vs. $\varepsilon^{48} \mathrm{Ti} \frac{47}{49}$ we using all $\mathrm{Ti}$ isotope dimensions. These plots show that the dominant sources present in the bulk meteorite data are not represented in the hibonite data as all hibonite component are resolved from both bulk meteorite components in both dimensions. (The only exception is the hibonite component $\{3\}$ in $\theta$ $\varepsilon^{50} \mathrm{Ti} \frac{47}{49}$ vs. $\varepsilon^{48} \mathrm{Ti} \frac{47}{49}$ which is very poorly defined and has essentially $100 \%$ uncertainties.) The errors are the $95 \%$ confidence intervals from the distribution of the bootstrapped ICA components. The dashed line in the inset figure is the lower error bound of the hibonite component $\{3\}$.

normal CAIs might be mixtures of the hibonite sources. Secondly, the hibonite population may represent more sources than the 4 to 6 that can be examined by a three dimensional
ICA. Lastly, a combination of these possibilities occurred and the 3 hibonite components represent more than 6 sources and that the bulk meteorites compositions are mixtures be- 


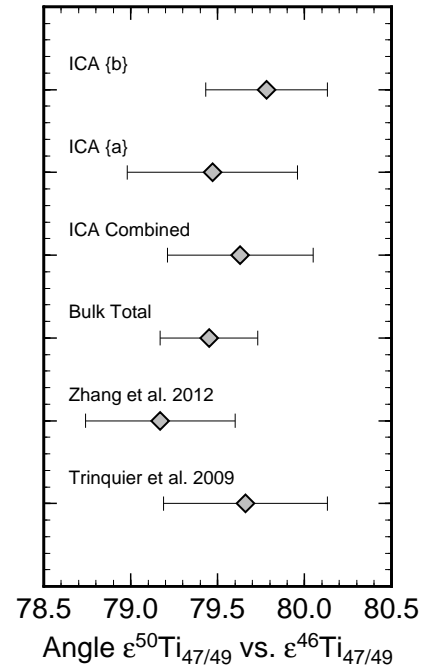

Fig. 3.- Figures a comparison between the estimates of angle of the bulk meteorite $\mathrm{Ti}$ isotope data in $\varepsilon^{50} \mathrm{Ti} \frac{47}{49}$ vs. $\varepsilon^{46} \mathrm{Ti} \frac{47}{49}$ estimated by York regression and ICA. The results of the two estimations are very similar which displays the power of ICA to estimate the slopes of correlations within data. ICA $\{a\}$ and $\mathrm{ICA}\{\mathrm{b}\}$ are the two components which are determined for the bulk meteorite population.

tween some of these sources. The likelihood and implications of these more complex scenarios are discussed below.

\subsection{Number of Sources}

The simplest explanation of the isotopic variation observed by the ICA is that the hibonites represent between 4 and 6 sources and the bulk meteorites and normal CAIs represent between 3 and 4 . These are the simplest explanations because they represent the fewest number of anomalous sources in the Solar System that can entirely explain the variation we observe. However, more complex models which involve more sources or mixing of sources are possible.

\subsubsection{Number of sources represented by the hibonites}

The hibonite system is under-constrained in that there are at least as many required components as dimensions. It is very difficult to examine how many components are required by an under-constrained system, and impossible to conclusively test. However, there are certain arguments and lines of evidence that suggest that the hibonite data, at the current level of precision, are only showing evidence for three components. Firstly, four sources can describe any three dimensional data array; akin to a point lying on a mixing line between two sources, there is no mathematical requirement for more sources to explain the spread of the data. Secondly, the components we have identified closely resemble the data with the largest variation is in $\gamma\left({ }^{50} \mathrm{Ti}_{\frac{47}{49}}\right)$ followed by $\gamma\left({ }^{46} \mathrm{Ti}_{\frac{47}{49}}\right)$ then $\gamma\left({ }^{48} \mathrm{Ti}_{49}^{49}\right)$. Moreover, the angles of these components fit with the angles of the original data such that the errors all but a few data overlap with the mixing vectors of the components, see figure 4 and further discussion in section 4.2.2. This demonstrates that the components are fully explaining the data without simply describing a cube around them. The most striking feature is that most data only require input from one component. Most hibonite data show evidence of binary mixing from one of three highly anomalous sources. Thirdly, the ICA tries to explain the variation in a dataset by making the sources as statistically independent as possible. Therefore, we can examine the possibility of unrepresented components by looking at the distribution of the three components we can identify.

As shown in figure 2, the vector of component $\{1\}$ is very different from the other two, this makes it very statistically resolved as this is a function of the angular separation and the distribution of point along the component vector. The angles of components $\{2\}$ and $\{3\}$ are much more similar. As the ICA hierarchically 
tries to assign components based on statistical significance, a 4th, unresolved component, must be at least as similar to one of the other components as $\{2\}$ and $\{3\}$ are to each other or else it would have been chosen. If a 4th component were to be present and unrepresented it would most likely lie in the plane of the $\{2\}$ and $\{3\}$ components as this is where several of the data which are not within error of a component vector reside. It is possible that if such a component exists it could be represented by the SHIB hibonite population, which are currently largely within error of terrestrial values.

A higher precision Ti isotope study of hibonite would significantly help resolve issues surrounding the number of sources. However, as the data stand with the PLAC population essentially showing all the variation, for the reasons described above, the most likely explanation is three components and four sources. We discuss the compositions of these sources in more detail below, see section 4.2 .

\subsubsection{Number of sources represented by the bulk meteorites}

The variation observed in the bulk meteorites is well described by two components, or at least three sources. This finding is in agreement with previous studies which show that there must be more than two sources in the bulk meteorite and normal CAI system (Trinquier et al. 2009; Williams et al. 2014). There is a hint in the bulk meteorite and normal CAI data that the third anomalous source is only present in the CAIs. This is because a York regression on the bulk meteorite population, without the normal CAI data, yield a slope of zero in $\varepsilon^{50} \mathrm{Ti}_{49} \frac{47}{49}$ vs. $\varepsilon^{48} \mathrm{Ti}_{49}$. Moreover, an ICA on the bulk meteorites alone shows no variation in the $\gamma\left({ }^{48} \mathrm{Ti}_{\frac{47}{49}}\right)$ dimension. This is supported by Williams et al. (2014) who have reported evidence for a third source in chondrules and CAIs in the absolute Ti isotope compositions. Absolute ratios include 


\subsection{Source Compositions}

Firstly, it is important to note that the ICA returns a vector along which the source composition lies. Therefore, it is not possible to say exactly what the source composition is, just the angle of its mixing line. However, by using the angles of the mixing lines we can identify compatible nucleosynthetic sources in the same way that York regressions on data have been used to examine nucleosynthetic sources in the past (e.g. Dauphas et al. 2004; Steele et al. 2012).

\subsubsection{Source compositions of bulk meteorite components}

The two components which the bulk meteorites and normal CAIs require are identical within error in $\gamma\left({ }^{50} \mathrm{Ti} \frac{47}{49}\right)$ vs. $\gamma\left({ }^{46} \mathrm{Ti}_{\frac{47}{49}}\right)$ and have angles identical to a York regression in the same dimensions on the original data of Trinquier et al. (2009), see figure 2 . This suggests binary mixing in $\varepsilon^{46} \mathrm{Ti}_{\frac{14}{49}}$ and $\varepsilon^{50} \mathrm{Ti}_{\frac{47}{49}}$ to produce this line. This source has been previously suggested to be input from the O/Ne zone of an SN II (Qin et al. 2011; Steele et al. 2012). The compositions produced by the $\mathrm{O} / \mathrm{Ne}$ zones of SN II vary with the mass of the pre supernova star. Few of the models of Rauscher et al. (2002) match exactly the slopes required to explain $\mathrm{Ti}$ isotope variation in the Solar System, they are however, all close. Titanium-50 and ${ }^{46} \mathrm{Ti}$ are thought to be dominantly produced in different nucleosynthetic environments. Therefore the finding that by including anomalies on the normalising isotopes the correct slopes of mixing may be produced is itself important. An alternative hypothesis to explain the meteorite correlation is simultaneous mixing of two distinct nucleosynthetic sources, one with $\varepsilon^{46} \mathrm{Ti} \frac{47}{49}$ anomalies and one with $\varepsilon^{50} \mathrm{Ti}_{\frac{47}{49}}$ anomalies, within the Solar System due to some common process (Trinquier et al. 2009). The ICA analysis is consistent with either interpretation.

In the other $\mathrm{Ti}$ axis, $\gamma\left({ }^{48} \mathrm{Ti}_{\frac{47}{49}}\right)$, however, there is slight variation between the two components. Note that the scale of the $\gamma\left({ }^{48} \mathrm{Ti}_{\frac{14}{49}}\right)$ axis on figure 2(c) significantly magnifies the apparent difference and the two components are in fact only divergent by 6 degrees. The component $\{a\}$ shows a positive correlation with an angle of $86.1_{-2.1}^{+1.2}$, while component $\{b\}$ is, in fact, almost vertical, so showing almost no influence on ${ }^{48} \mathrm{Ti}$, with an angle of $92.3_{-1.5}^{+4.7}$. The positive correlation in component $\{a\}$ is just visible in the original meteorite and normal CAI data, see figure 1(d). However, the apparent correlation is only present in the CAI data. This may suggest that the CAIs are sampling a source which imparts a very slight anomaly in $\varepsilon^{48} \mathrm{Ti}_{\frac{47}{49}}$ and is not present in the bulk meteorite population. This inference may be supported by recent absolute $\mathrm{Ti}$ isotope measurements of Williams et al. (2014) who found evidence for correlated ${ }^{46} \mathrm{Ti},{ }^{47} \mathrm{Ti}$ and ${ }^{50} \mathrm{Ti}$ anomalies in the bulk meteorites while the CAIs plotted off this trend in ${ }^{47} \mathrm{Ti}$. It is not clear if this can explain the deviation in $\varepsilon^{48} \mathrm{Ti}_{\frac{47}{49}}$ observed in the previous measurements, but is supporting evidence that the CAIs may be sampling a subtly different set of sources. An ICA performed on the bulk meteorites alone yields one component identical in angle in $\gamma\left({ }^{50} \mathrm{Ti} \frac{47}{49}\right)$ vs. $\gamma\left({ }^{46} \mathrm{Ti}_{49}^{47}\right)$ to the two component ICA and with zero variation in $\gamma\left({ }^{48} \mathrm{Ti}_{\frac{47}{49}}\right)$ which may support this hypothesis further. The major conclusions of this study are not affected by this debate because the variation in $\gamma\left({ }^{50} \mathrm{Ti} \frac{47}{49}\right)$ vs. $\gamma\left({ }^{46} \mathrm{Ti} \frac{47}{49}\right)$ is not affected and the variation in $\gamma\left({ }^{48} \mathrm{Ti} \frac{47}{49}\right)$ is very small and always resolved from the hibonite components. However, the hint that the CAIs, while dominated by the same anomalous sources as the bulk meteorites, may sample an additional anomalous source is very interesting. This finding could have significant implications for early Solar System mixing and warrants further study. 


\subsubsection{Source compositions of the hibonite} components

The nucleosynthetic origins of the hibonite sources have been less well studied in a quantitative sense and the overall lack of precision in the components we have identified does not makes this endeavour much easier. Interestingly, the errors of most of the hibonite grains overlap with at least one of the identified components, for example in $\varepsilon^{50} \mathrm{Ti}_{49}^{47}$ vs. $\varepsilon^{46} \mathrm{Ti} \frac{47}{49}$ see figure 4 . This suggests the hibonite grains may mostly be exhibiting binary mixing to one anomalous nucleosynthetic source. In the rare cases of the remaining data which lie a long way from any of the component vectors, these compositions may be produced by mixing two of the anomalous nucleosynthetic sources. Mixing two sources is not an unexpected result as the ICA is designed to deconvolve multivariate data. Even so, a finding of binary mixing in the majority of the hibonites presents a simplified case which may offer more easily testable predictions for mixing in other isotope systems.

As discussed above, the components of the hibonites are not equally well defined. The most precisely defined component is component $\{1\}$ with a angle of $88.7_{-1.1}^{+1.0}$ in $\gamma\left({ }^{50} \mathrm{Ti}_{49}\right)$ vs. $\gamma\left({ }^{46} \mathrm{Ti}_{47} \frac{47}{49}\right)$ and $91.0_{-0.5}^{+0.5}$ in $\gamma\left({ }^{50} \mathrm{Ti}_{\frac{47}{49}}\right)$ vs. $\gamma\left({ }^{48} \mathrm{Ti} \frac{47}{49}\right)$. These angles may be well enough defined to examine the nucleosynthetic significance, however, it is not clear where these signatures may be produced.

As with previous studies (e.g. Steele et al. 2012) we are looking for a signature that describes the characteristic of a distinct nucleosynthetic environment. The most significant finding is a feature which is consistent among most, if not all, models. For example the ${ }^{58} \mathrm{Ni}$ anomaly found in the $\mathrm{Si} / \mathrm{S}$ zone of all masses SN II (Steele et al. 2012). We have compared the slopes of the hibonite components to the same range of supernova models as previous studies: for type Ia supernova (SN Ia), Woosley (1997); Travaglio et al.
(2004); Iwamoto et al. (1999); Hashimoto (1995); Maeda et al. (2010); Travaglio et al. (2011); for SN II, Iwamoto et al. (1999); Umeda and Nomoto (2002); Nomoto et al. (1997); Rauscher et al. (2002); Hashimoto (1995); for asymptotic giant branch star (AGB), models were kindly provided by Gallino and Davis (pers. comm. 2009); and for individual shells of SN II we used models from Meyer et al. (1995) Rauscher et al. (2002). Bulk homogenous SN II are not a likely candidate, both because it is unrealistic they would present a single homogenised signature, and also this signature does not produce the high $\varepsilon^{50} \mathrm{Ti}_{49} \frac{47}{4}$ anomalies required. Large ${ }^{50} \mathrm{Ti}$ excesses are produced in the inner regions of SN II suggesting this as a possible candidate. However, these excesses are accompanied with significant deficits in ${ }^{47} \mathrm{Ti}$ which in the ${ }^{47} \mathrm{Ti} /{ }^{49} \mathrm{Ti}$ normalization acts to reduce the apparent ${ }^{50} \mathrm{Ti}$ anomaly, and increase the apparent ${ }^{46} \mathrm{Ti}$ anomaly, such that the slope in $\varepsilon^{50} \mathrm{Ti}_{\frac{47}{49}}$ vs. $\varepsilon^{46} \mathrm{Ti}_{\frac{47}{49}}$ is always too low. Finally, large excesses in ${ }^{50} \mathrm{Ti}$, not accompanied by excesses of ${ }^{47} \mathrm{Ti}$ or ${ }^{46} \mathrm{Ti}$, are a general, if not universal, feature of SN Ia. This suggestion is further supported by the large positive ${ }^{48} \mathrm{Ca}$ anomalies which are correlated with ${ }^{50} \mathrm{Ti}$ anomalies (e.g. Zinner et al. 1986). Large ${ }^{48} \mathrm{Ca}$ anomalies, in absence of ${ }^{46} \mathrm{Ca}$ anomalies, are strong evidence for input from an SN Ia (Meyer et al. 1996). This possibly makes SN Ia the most likely source for the high ${ }^{50} \mathrm{Ti}$ component in the hibonites, but this is by no means conclusive.

The other two components are not nearly as well defined and no nucleosynthetic significance can be determined due to the large uncertainties on their compositions. One of the most intriguing aspects of the hibonite grains is that they have both large positive and large negative anomalies in for example, $\varepsilon^{50} \mathrm{Ti}_{\frac{47}{49}}$, see figure 1. These components represent the sources of hibonite grains which exhibit negative anomalies in $\varepsilon^{50} \mathrm{Ti} \frac{47}{49}$ and $\varepsilon^{46} \mathrm{Ti}_{49}$. These large positive and negative anomalies are dif- 
ficult to reconcile with the very small range in bulk meteorites in the context binary mixing. However, in the context of binary mixing between multiple sources these anomalies are more easily understood. Broadly, component $\{2\}$ constitutes low $\gamma\left({ }^{50} \mathrm{Ti} \frac{47}{49}\right)$, slight $\gamma\left({ }^{46} \mathrm{Ti} \frac{47}{49}\right)$ and high $\gamma\left({ }^{48} \mathrm{Ti}_{\frac{47}{49}}\right)$, while component $\{3\}$ is made up of low $\gamma\left({ }^{50} \mathrm{Ti}_{\frac{47}{49}}\right)$, high $\gamma\left({ }^{46} \mathrm{Ti} \frac{47}{49}\right)$ and low $\gamma\left({ }^{48} \mathrm{Ti}_{49}^{47}\right)$. These two components are not well defined enough to quantitatively discuss the nucleosynthetic significance, however, qualitative assessments may be made. Component $\{2\}$ is consistent with input from material from low mass $(\sim 13-15$ $\left.\mathrm{M}_{\odot}\right)$ SN II models, while the sources of component $\{3\}$ are consistent with several midmass $\left(\sim 25-30 \quad \mathrm{M}_{\odot}\right)$ SN II (Hashimoto 1995; Umeda and Nomoto 2002). Even though the sources cannot be definitively identified, these components are still useful for discussing potential mixing environments within the Solar System as they are helpful for interpreting the hibonite data, see section 4.3

\subsection{Implications for mixing and sources within the Solar System}

The ICA of the hibonite data returns the compositions of three components which most likely reflect mixing of four sources. Most of the data cluster around zero, which suggests that one of the sources is represented by this composition. Interestingly, this composition is compatible with the composition of one of the bulk meteorite sources. This may be close in composition one of the end of the bulk meteorite correlation and could represent the bulk composition of the presolar molecular cloud, see section 4.3.1 below. That one of the sources of the two different sample sets may be the same is interesting and also may present a more likely scenario because it reduces the number of required sources. However, we must examine the likelihood that such a source may exist.

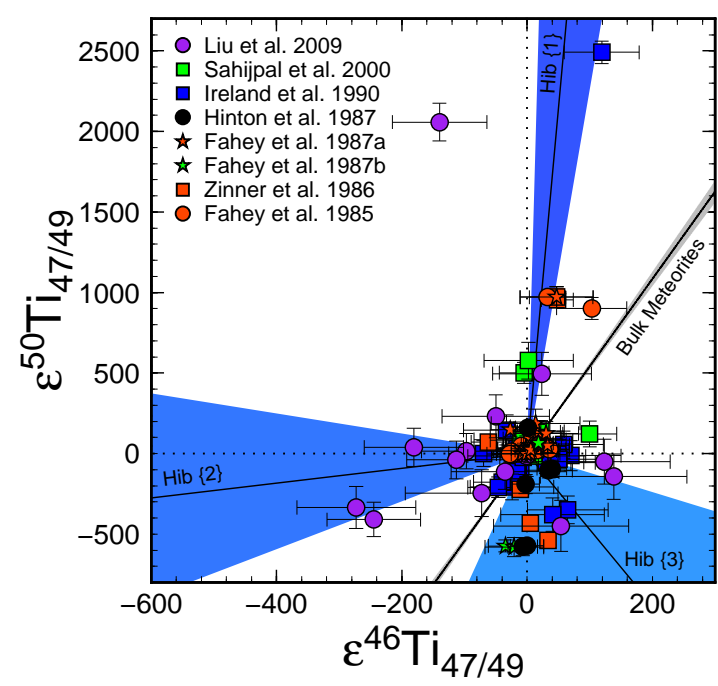

Fig. 4.- Figures showing the Ti isotope compositions for hibonite grains and the components determined from the ICA. The data, with only a few exceptions, are within error of one of the components. This suggests that there is no requirement for a further components. The compositions of few point that lie outside of error of all the components can be created by mixing two of the components.

\subsubsection{Material in proto-planetary disks, molecular clouds and the interstellar medium}

The identity of the material of this shared composition is likely the average of highly processed material from the parent molecular cloud. The journey of stellar condensates from stars and supernovae to proto-planetary disks is not a simple one. There are many stages of processing and mechanisms by which they may be altered and destroyed.

The Solar System, and proto-planetary disks in general, form from a mixture of material from many different generations of stars likely integrating material over billions of years of galactic evolution. Indeed, it has been estimated that the timescale for a grain from a stellar sources to traverse the interstellar medium (ISM) and be incorporated into a proto-planetary disk is on the order of a $1 \mathrm{Ga}$ (Dwek and Scalo 1980; Jones and Nuth 2011). 
However, due to physical processing and irradiation in the interstellar medium it is an oversimplification to assume that the material in a molecular cloud contains the original isotopic signatures of stars integrated over 1 or 2 Ga.

Material in molecular clouds may have experienced several cycles through the diffuse interstellar medium into molecular clouds without necessarily being incorporated into a proto-planetary disk, or a star, to be reprocessed. Lifetimes of refractory grains have been estimated at $4 \times 10^{8}-20 \times 10^{8}$ years depending on how much time the grains spend in molecular clouds where stars are more likely to form (see, Barlow 1978; Jones 2004, for discussions). Interestingly, Molster and Waters (2003) note that crystalline silicates are only observed around supernovae and young stars and proto-planetary disks - where they are likely to be forming. This suggests from an observational standpoint that fresh material does not last long in the ISM. Carrez et al. (2002) performed laboratory experiments to investigate the effects of He irradiation of olivine as an analogue to radiation processing in interstellar medium finding that both chemical and structural changes occur in olivine culminating in amorphitization. While, from astronomical observations, Kemper et al. (2004) find that the timescale of amorphitization in the ISM is on the order of 5-9 Ma.

Therefore, in most cases the lifetime of grains will be less than the timescale from production in supernova to processing in protoplanetary disk. It follows, then, that many grains will not survive their journey through the interstellar medium to be incorporated into a proto-planetary disk. Rather these grains will become amorphitized and eventually destroyed. This material will remain in the ISM and form new grains. Jones and Nuth (2011) estimate that the majority (90-95\%) of grains observed in the ISM would have formed in in situ and so will not represent pure stellar compositions. The action of this grain reformation will be to homogenise the isotopic compositions of the many generations and types of stars that have given material to the ISM. From this argument alone it seems logical that material from the majority of old stellar sources will have been homogenised to yield a single, averaged, composition in the early Solar System and not a multitude of grains with highly exotic compositions integrating stellar sources over billions of years. Moreover, as Molster and Waters (2003) describe, crystalline grains are only observed around young stars (proto-planetary disks) and evolved stars (e.g. winds from AGB) but not in the ISM. This suggests that the grains that are forming in the ISM are amorphous and may be more volatile and so may have been more easily further homogenised by higher temperature processing in the proto-Solar nebula.

Younger grains from more recent events ( $\ll 1 \mathrm{Ga}$ to $\sim 1$ Ma prior to the formation of the proto-Solar nebula), however, are more likely to survive intact and to remain crystalline. Therefore, more recent events may still present grains with large anomalies to the early Solar System. Due to the lifetime of grains $\left(5-20 \times 10^{8}\right.$ years, Barlow 1978) it is unlikely they will have been destroyed to form new phases so will not reflect the homogenised isotopic compositions of the host molecular cloud or ISM. However, to a variable degree they will likely have experienced processing in the ISM or molecular cloud and this may affect their ability to contribute to isotopic heterogeneity in the proto-planetary disk.

An important point to clarify here is what the anomalies that are measured mean in terms of grains, or carrier phases. To present an isotopic anomaly in the early Solar System, grains must be heterogeneously distributed. For example, large anomalies are present in $\mathrm{CC}$ leachates in $\mathrm{Zr}$ isotopes (Schönbächler 
et al. 2005), however, much smaller anomalies are present in the bulk meteorites. This is because the carrier phase of $\mathrm{Zr}$ isotopes anomalies was relatively homogeneously distributed between the different chondrite parent bodies. For the bulk meteorites, normal CAI and hibonite populations of interest to this study, the anomalies represent different abundances of carrier phases. Therefore, the effectiveness of a grain to transfer an anomaly from a stellar source to a proto-planetary disk relies on its ability to either remain, or become, heterogeneously distributed through the nebula. Factors that may affect this are: volatility, as volatile grains are effectively mixed as they enter the gas phase; crystallinity, as more crystalline phases may be more refractory but also effectively sorted by physical processes such as photophoresis (Krauss and Wurm 2005; Wurm et al. 2010); size; density or chemistry. Clearly some of the factors are more important than others; if the grains are chemically unstable or volatile, size and density sorting will be less significant. Therefore, we can examine the effects of processing in the molecular cloud and ISM on some of these important factors for the preservation of anomalies.

\subsubsection{Relationships between hibonite and bulk meteorite sources}

We find that the sources of the hibonite $\mathrm{Ti}$ isotope anomalies are not related to the sources of the bulk meteorite anomalies, see section 3.1 above. This is an interesting finding because while the hibonites represent the largest Ti isotope anomalies in the Solar System, the bulk meteorites constitute much more material and so actually contain a much greater mass of anomalous Ti. Therefore, it might have been expected that these two repositories of anomalous $\mathrm{Ti}$ share a common source.

There are two end-member explanations for why the hibonite sources and the bulk meteorites sources are not the same:
1. The dominant source in the bulk meteorites is not a primary nucleosynthetic source(s) as previously thought (e.g. Leya et al. 2008, 2009; Qin et al. 2011; Steele et al. 2012) but rather is made within the Solar System by mixing one or more of the hibonite sources, or some other sources, at some point after hibonite formation but before CAI formation as normal CAIs sample the same sources as the bulk meteorites (Trinquier et al. 2009).

2. The hibonite sources, while highly concentrated in the hibonite forming regions, are not dominant in the Solar System and have been diluted to extinction during subsequent mixing.

The first scenario poses the very interesting prospect of being able to directly examine the timescales of early Solar System mixing in the early Solar System. By dating hibonite formation we may find the final time at which primary nucleosynthetic sources were sampled. This may then be compared with the age of CAI formation, after which time full Solar System mixing of the primary sources had occurred. This comparison will yield information about the time taken for large scale homogenisation of the primary nucleosynthetic sources. As discussed above, and in previous studies (e.g. Ireland 1990), the hibonites with the largest anomalies are the PLACs, these then might represent the earliest samples. The SHIBs have much smaller, or no, stable isotope anomalies coupled with variable ${ }^{26} \mathrm{Al}$ abundances (Sahijpal and Goswami 1998). The SHIBs may simply represent hibonite formation contemporaneously with the PLACs, but in a region with lower abundance of anomalous $\mathrm{Ti}$ carriers and higher abundances of ${ }^{26} \mathrm{Al}$ carriers (Liu et al. 2012). Alternatively, the SHIBs may represent an intermediate stage of mixing between the PLAC sources and the bulk meteorite sources. Possibly the carrier phase for the bulk meteorite 
variation.

While it is certainly a possibility that the SHIBs represent an intermediate stage of mixing, the current level of precision on $\mathrm{Ti}$ isotope compositions the hibonite population are not high enough to determine if this is the case. However, we can place limits on the extent of mixing that would have had to occur. The precision of the hibonite measurements would place the bulk meteorite source composition only $+200 \%$ in $\varepsilon^{50} \mathrm{Ti}_{49} \frac{47}{49}$ and +50 $\% 00$ in $\varepsilon^{46} \mathrm{Ti}_{49} \frac{47}{49}$ In order to create the variation in $\varepsilon^{50} \mathrm{Ti}_{49}^{47}$ from SHIBs with this composition would require hibonite abundances between 50 and 200 times those observed in CM hibonites, depending on $\mathrm{Ti}$ concentration in the hibonites (using concentration and abundance data from Ireland et al. 1988; Wasson and Kallemeyn 1988). Under nebula conditions, outside the CAI forming region, hibonite is a physically, chemically and thermally stable mineral, therefore, if hibonite grains had been present in these abundances it seems unlikely it would been depleted so significantly in all chondrite groups, especially in primitive groups such as CM chondrites. Moreover, other chondrites contain even lower abundances of hibonite, so would require even more complete reprocessing. Therefore, we favor the second model in which the hibonite sources, although highly anomalous in the hibonite forming region, are not dominant on a bulk Solar System scale (e.g. Boss 2008).

The second scenario, in which the sources hibonite of $\mathrm{Ti}$ isotope anomalies are diluted to extinction during the final stages of homogenisation in the protosolar nebula, makes the hibonite source compositions less significant for the bulk composition of the Solar System. However, these sources record nucleosynthetic information not present in other early Solar System samples and so may provide a tantalising, fine scale, view of the astrophysical birth environment of the Solar System. They may record the compositions of stellar environments which, while not dominant, contributed material to the Solar System while it was forming; they provide a more complete view of all of the stellar sources to the protoSolar Nebula.

The sources hibonite $\mathrm{Ti}$ isotope anomalies were not homogenised into the molecular cloud composition, therefore they must be younger than around $100 \mathrm{Ma}$, see above section 4.3.1. These sources were likely some combination of SN Ia and SN II. The grains in material ejected during these events would still be crystalline after $\sim 100 \mathrm{Ma}$ and so would have remained relatively impervious to thermal processing during the early collapse of the proto-Solar nebula. This relatively recently synthesised material, may have been concentrated in clumps as it hasn't had as much time to processed and mixed into the molecular cloud, so could survive in relatively concentrated clumps in the proto-solar nebula. These clumps may be distributed throughout the proto-solar nebula, but the only samples we derive form this time were in the hibonite forming region. It is in these clumps that the hibonites would form, partially mixing with homogenised molecular cloud material to give the compositions we observe in the hibonites today. Subsequently, these clumps would be dynamically mixed into the rest of the Solar System (e.g. Boss 2008; Ciesla 2009).

There are three mechanisms by which the highly anomalous material in these clump would not dominate the bulk meteorite compositions which formed later. Firstly, the sources may have been in such low concentration in the bulk proto-solar nebula that they were simply be diluted to extinction. Secondly, they may have been somewhat amorphitized by radiation damage in the molecular cloud and more susceptible to the thermal processing than the bulk meteorite source. Lastly, the majority of the material may have been lost to the proto-Sun during infall and that the hibonite grains were propelled back out 
into the Solar System on stellar winds or in 1250 bipolar outflow jets (e.g. Shu et al. 2001).

In addition to large stable isotope anomalies, the PLACs, which best represent the hibonite sources, are devoid of evidence for live ${ }^{26} \mathrm{Al}$. Therefore, the hibonites may have formed from material devoid of ${ }^{26} \mathrm{Al}$, either dominantly form a supernova zone which does not produce ${ }^{26} \mathrm{Al}$ or from material older than $\gtrsim 5$ Ma. A second possibility is that the hibonite grains formed contemporaneously with CAIs and experienced a thermal event in the Solar System $\sim 5$ Ma after CAI formation which would have erased any signature of ${ }^{26} \mathrm{Al}$. However, if the hibonite and normal population of CAIs formed contemporaneously it is difficult understand why the hibonite grains have such large stable isotope anomalies.

\subsubsection{Why is the bulk meteorite source dom- inant?}

There is evidence that a wide variety of different nucleosynthetic sources were present in the proto-Solar nebula. However, only one of these sources dominates the Ti isotope variation in the bulk meteorites. Taking again the example of $\mathrm{Ti}$, the $\mathrm{O} / \mathrm{Ne}$ zone from a $\mathrm{SN}$ II appears to be the dominant source in the bulk meteorites, and so the bulk Solar System. It is possible that this source is dominant simply because it was the most concentrated. However, due to processing in the ISM, material from older stellar sources may be more readily homogenised in the early Solar system, see section 4.3.1 above. In this way material from more recent nucleosynthetic sources may be more resistant to mixing in the early Solar System. Therefore, the dominant sources in the bulk meteorites and normal CAIs may not be the most concentrated but the freshest, or more recently synthesised. With further studies it may be possible to test this hypothesis by examining potential correlations between the sources of stable isotope anomalies (like $\mathrm{Ti}$ ) and the sources of SLRs (like ${ }^{26} \mathrm{Al}$ ).

\section{Conclusions}

We have examined mixing relationships in the early Solar System by comparing the sources of $\mathrm{Ti}$ isotope variation. Using Independent Component Analysis we have compared the sources of $\mathrm{Ti}$ isotope variation in hibonite grains and bulk meteorites. We find the variation in bulk meteorites represents mixing of three sources, while the hibonite populations are consistent with mixing of four sources. The ICA shows that the highly anomalous sources of bulk meteorite and hibonite $\mathrm{Ti}$ isotope variation are not related. One source which is consistent between both data sets has a composition close to the terrestrial ratio. This source may be represented by molecular cloud material, highly processed and homogenised by radiation and thermal events significantly before the start of the Solar System.

The finding that the anomalous nucleosynthetic sources represented by the variation in hibonite and bulk meteorites are not related has significant implications for mixing in the early Solar System. One possible explanation is that the sources which dominate the variation in the bulk meteorites are not primary nucleosynthetic sources but rather were created by mixing two or more hibonite sources. However, this requires a stage of full protoSolar nebula mixing from which we derive no samples. An alternative hypothesis is that the hibonite sources, although dominant in the hibonite forming regions, are not significant sources for isotope anomalies in the bulk Solar System. This may be because the sources were of low concentration after major homogenisation had occurred. Another, possibly more likely, hypothesis is that is that the carrier phases of the hibonite $\mathrm{Ti}$ isotope anomalies, while abundant, were not physically or chemically robust enough to survive the vigorous mixing in the proto-Solar nebula. 


\section{Acknowledgements}

We would like to thank Kevin McKeegan and Ming-Chang Liu for detailed and insightful comments, thorough discussion and encouragement. We would also like to thank Andrew Davis and Levke Kööp for reviewing this manuscript and their detailed comments which greatly improved the manuscript. We would like to thank Eric Feigelson for his editorial handling. This work was funded by UCLA and NASA Cosmochemistry.

\section{REFERENCES}

Barlow, M. J., 1978. The destruction and growth of dust grains in interstellar space. I - Destruction by sputtering. Monthly Notices of the Royal Astronomical Society 183, 367-395.

Boss, A. P., 2008. Mixing in the solar nebula: Implications for isotopic heterogeneity and large-scale transport of refractory grains. Earth and Planetary Science Letters 268 (1-2), 102-109.

Burkhardt, C., Kleine, T., Oberli, F., Pack, A., Bourdon, B., Wieler, R., 2011. Molybdenum isotope anomalies in meteorites: Constraints on solar nebula evolution and origin of the earth. Earth and Planetary Science Letters 312 (3-4), 390-400.

Carrez, P., Demyk, K., Cordier, P., Gengembre, L., Grimblot, J., D'Hendecourt, L., Jones, A. P., Leroux, H., 2002. Low-energy helium ion irradiation-induced amorphization and chemical changes in olivine: Insights for silicate dust evolution in the interstellar medium. Meteoritics \& Planetary Science 37 (11), 1599-1614.

Ciesla, F. J., 2009. Two-dimensional transport of solids in viscous protoplanetary disks. Icarus 200 (2), 655-671.

Comon, P., 1994. Independent component analysis, a new concept? Signal Processing36 (3), 287-314.

Cuesta-Albertos, J., Gordaliza, A., Matrán, C., et al., 1997. Trimmed $k$-means: an attempt to robustify quantizers. The Annals of Statistics 25 (2), 553-576.

Dauphas, N., Cook, D. L., Sacarabany, A., Fröhlich, C., Davis, A. M., Wadhwa, M., Pourmand, A., Rauscher, T., Gallino, R., 2008. Iron-60 Evidence for Early Injection and Efficient Mixing of Stellar Debris in the Protosolar Nebula. The Astrophysical Journal 686 (1), 560-569.

Dauphas, N., Davis, A., Marty, B., Reisberg, L., 2004. The cosmic molybdenumruthenium isotope correlation. Earth and Planetary Science Letters 226 (3), 465-475.

Dauphas, N., Remusat, L., Chen, J. H., Roskosz, M., Papanastassiou, D. A., Stodolna, J., Guan, Y., Ma, C., Eiler, J. M., 2010. Neutron-rich Chromium Isotope Anomalies in Supernova Nanoparticles. The Astrophysical Journal 720, 15771591.

Dwek, E., Scalo, J. M., 1980. The evolution of refractory interstellar grains in the solar neighborhood. The Astrophysical Journal 239, 193-211.

Efron, B., 1981. Nonparametric estimates of standard error: the jackknife, the bootstrap and other methods. Biometrika 68 (3), 589599 .

Fahey, A., Goswami, J. N., McKeegan, K. D., Zinner, E., 1985. Evidence for extreme Ti50 enrichments in primitive meteorites. The Astrophysical Journal, Letters 296, L17L20.

Fahey, A. J., Goswami, J. N., McKeegan, K. D., Zinner, E. K., 1987a. ${ }^{26} \mathrm{Al},{ }^{244} \mathrm{Pu}$, ${ }^{50} \mathrm{Ti}, \mathrm{REE}$, and trace element abundances 
in hibonite grains from $\mathrm{CM}$ and $\mathrm{CV}$ meteorites. Geochimica et Cosmochimica Acta 51 (2), 329-350.

Fahey, A. J., Goswami, J. N., McKeegan, K. D., Zinner, E. K., 1987b. O-16 excesses in Murchison and Murray hibonites - A case against a late supernova injection origin of isotopic anomalies in $\mathrm{O}, \mathrm{Mg}, \mathrm{Ca}$, and Ti. The Astrophysical Journal, Letters 323, L91-L95.

Fritz, H., Garcia-Escudero, L. A., Mayo-Iscar, A., 2012. tclust: An r package for a trimming approach to cluster analysis. Journal of Statistical Software 47 (12), 1-26.

Gallino, R., Davis, A., 2009. Personal communication.

Hashimoto, M., 1995. Supernova nucleosynthesis in massive stars. Progress of Theoretical Physics 94 (5), 663-736.

Heydegger, H. R., Foster, J. J., Compston, W., 1979. Evidence of a new isotopic anomaly from titanium isotopic ratios in meteoric materials. Nature 278 (5706), 704707.

Hinton, R. W., Davis, A. M., Scatena-Wachel, D. E., 1987. Large negative Ti-50 anomalies in refractory inclusions from the Murchison carbonaceous chondrite - Evidence for incomplete mixing of neutron-rich supernova ejecta into the solar system. The Astrophysical Journal 313, 420-428.

Hyvarinen, A., 1999. Fast and robust fixedpoint algorithms for independent component analysis. Neural Networks, IEEE Transactions on 10 (3), 626-634.

Hyvärinen, A., Oja, E., 2000. Independent component analysis: algorithms and applications. Neural Networks13 (4-5), 411-430.

Ireland, T. R., 1990. Presolar isotopic and chemical signatures in hibonite-bearing re- fractory inclusions from the Murchison carbonaceous chondrite. Geochimica et Cosmochimica Acta 54 (11), 3219-3237.

Ireland, T. R., Fahey, A. J., Zinner, E. K., 1988. Trace-element abundances in hibonites from the murchison carbonaceous chondrite: Constraints on hightemperature processes in the solar nebula. Geochimica et Cosmochimica Acta 52 (12), 2841-2854.

Ireland, T. R., Zinner, E. K., Fahey, A. J., Esat, T. M., 1992. Evidence for distillation in the formation of hal and related hibonite inclusions. Geochimica et Cosmochimica Acta 56 (6), 2503-2520.

Iwamori, H., Albarède, F., 2008. Decoupled isotopic record of ridge and subduction zone processes in oceanic basalts by independent component analysis. Geochemistry, Geophysics, Geosystems9 (4).

Iwamoto, K., Brachwitz, F., Nomoto, K., Kishimoto, N., Umeda, H., Hix, W. R., Thielemann, F., 1999. Nucleosynthesis in chandrasekhar mass models for type IA supernovae and constraints on progenitor systems and burning-front propagation. Astrophysical Journal, Supplement Series 125, 439-462.

Jones, A. P., May 2004. Dust Destruction Processes. In: Witt, A. N., Clayton, G. C., Draine, B. T. (Eds.), Astrophysics of Dust. Vol. 309 of Astronomical Society of the Pacific Conference Series. p. 347.

Jones, A. P., Nuth, J. A., 2011. Dust destruction in the ism: a re-evaluation of dust lifetimes. A\&A 530.

Kemper, F., Vriend, W. J., Tielens, A. G. G. M., 2004. The absence of crystalline silicates in the diffuse interstellar medium. The Astrophysical Journal 609 (2), 826-837. 
Krauss, O., Wurm, G., 2005. Photophoresis and the pile-up of dust in young circumstellar disks. The Astrophysical Journal $630(2), 1088$.

Lee, T., Papanastassiou, D. A., Wasserburg, G. J., 1978. Calcium isotopic anomalies in the Allende meteorite. The Astrophysical Journal, Letters 220, L21-L25.

Leya, I., Schönbächler, M., Wiechert, U., Krähenbühl, U., Halliday, A. N., 2008. Titanium isotopes and the radial heterogeneity of the solar system. Earth and Planetary Science Letters 266, 233-244.

Leya, I., Schönbächler, M., Krähenbühl, U, Halliday, A. N., 2009. New Titanium Isotope Data for Allende and Efremovka CAIs. The Astrophysical Journal 702 (2), 1118 1126.

Liu, M.-C., Chaussidon, M., Göpel, C., Lee, T., 2012. A heterogeneous solar nebula as sampled by $\mathrm{cm}$ hibonite grains. Earth and Planetary Science Letters 327-328 (0), 7583.

Liu, M.-C., McKeegan, K. D., Goswami, J. N., Marhas, K. K., Sahijpal, S., Ireland, T. R., Davis, A. M., 2009. Isotopic records in $\mathrm{cm}$ hibonites: Implications for timescales of mixing of isotope reservoirs in the solar nebula. Geochimica et Cosmochimica Acta 73 (17), 5051-5079.

Lyons, J., Young, E., 2005. CO self-shielding as the origin of oxygen isotope anomalies in the early solar nebula. Nature 435 (7040), 317-20.

Lyons, J. R., Bergin, E. A., Ciesla, F. J., Davis, A. M., Desch, S. J., Hashizume, K., Lee, J. E., 9 2009. Timescales for the evolution of oxygen isotope compositions in the solar nebula. Geochimica et Cosmochimica Acta 73 (17), 4998-5017.
Maeda, K., Röpke, F. K., Fink, M., Hillebrandt, W., Traviglio, C., Thielemann, F., 2010. Nucleosynthesis in two-dimensional delayed detonation models of type Ia supernova explosions. The Astrophysical Journal 712 (1), 624-638.

Mahon, K., 1996. The new "York" regression: Application of an improved statistical method to geochemistry. International Geology Review 38 (4), 293-303.

Marchini, J., Heaton, C., Ripley, B., 2013. fastica: Fastica algorithms to perform ica and projection pursuit. $\mathrm{R}$ package version, 1-2.

Marhas, K. K., Goswami, J. N., Davis, A. M., 2002. Short-lived nuclides in hibonite grains from murchison: Evidence for solar system evolution. Science 298 (5601), 2182-2185.

McCulloch, M. T., Wasserburg, G. J., 1978. Barium and neodymium isotopic anomalies in the Allende meteorite. The Astrophysical Journal, Letters 220, L15-L19.

Meyer, B. S., Krishnan, T. D., Clayton, D. D., 1996. ${ }^{48} \mathrm{Ca}$ production in matter expanding from high temperature and density. The Astrophysical Journal 462, 825-839.

Meyer, B. S., Weaver, T. A., Woosley, S. E., 1995. Isotope source table for a $25 \mathrm{M}_{\odot}$ supernova. Meteoritics 30, 325-334.

Molster, F., Waters, L., 2003. The mineralogy of interstellar and circumstellar dust. In: Henning, T. (Ed.), Astromineralogy. Vol. 609 of Lecture Notes in Physics. Springer Berlin Heidelberg, pp. 121-170.

Niederer, F. R., Papanastassiou, D. A., Wasserburg, G. J., 1985. Absolute isotopic abundances of Ti in meteorites. Geochimica et Cosmochimica Acta 49 (3), 835-851.

Niemeyer, S., Lugmair, G. W., 1980. Ti isotope anomalies in an "un-fun" Allende inclusion. Meteoritics 15 (4), 341. 
Niemeyer, S., Lugmair, G. W., 1981. Ubiquitous isotopic anomalies in $\mathrm{Ti}$ from normal Allende inclusions. Earth and Planetary Science Letters 53 (2), 211-225.

Nomoto, K., Hashimoto, M., Tsujimoto, T., Thielemann, F. K., Kishimoto, N., Kubo, Y., Nakasato, N., 1997. Nucleosynthesis in type II supernovae. Nuclear Physics A 616, 79-90.

Qin, L., Nittler, L. R., Alexander, C. M. O., Wang, J., Stadermann, F. J., Carlson, R. W., 2011. Extreme ${ }^{54} \mathrm{Cr}$-rich nanooxides in the CI chondrite Orgueil Implication for a late supernova injection into the Solar System. Geochimica et Cosmochimica Acta 75 (2), 629-644.

R Core Team, 2013. R: A Language and Environment for Statistical Computing. R Foundation for Statistical Computing, Vienna, Austria.

Rauscher, T., Heger, A., Hoffman, R. D. Woosley, S. E., 2002. Nucleosynthesis in Massive Stars with Improved Nuclear and Stellar Physics. The Astrophysical Journal 576 (1), 323-348.

Russell, W., Papanastassiou, D., Tombrello, T., 1978. Ca isotope fractionation on the Earth and other Solar System materials. Geochimica et Cosmochimica Acta 42, 1075-1090.

Sahijpal, S., Goswami, J. N., 1998. Refractory phases in primitive meteorites devoid of 26al and 41ca:representative samples of first solar system solids? The Astrophysical Journal Letters 509 (2), L137.

Sahijpal, S., Goswami, J. N., Davis, A. M., 2000. K, Mg, Ti and Ca isotopic compositions and refractory trace element abundances in hibonites from CM and CV meteorites: implications for early solar system processes. Geochimica et Cosmochimica Acta 64 (11), 1989-2005.
Schönbächler, M., Rehkämper, M., Fehr, M. A., Halliday, A. N., Hattendorf, B., Günther, D., 2005. Nucleosynthetic zirconium isotope anomalies in acid leachates of carbonaceous chondrites. Geochimica et Cosmochimica Acta 69 (21), 5113-5122.

Shu, F., Shang, H., Gounelle, M., Glassgold, A., Lee, T., 2001. The origin of chondrules and refractory inclusions in chondritic meteorites. The Astrophysical Journal 548 (2), 1029-1050.

Steele, R. C. J., Coath, C. D., Regelous, M., Russell, S., Elliott, T., 2010. Correlated Neutron Rich Ni Isotope Anomalies in Chondritic and Iron Meteorites. Lunar and Planetary Institute Science Conference Abstracts 41, 1984.

Steele, R. C. J., Coath, C. D., Regelous, M., Russell, S., Elliott, T., 2012. Neutron-poor nickel isotope anomalies in meteorites. The Astrophysical Journal 758 (1), 59.

Steele, R. C. J., Elliott, T., Coath, C. D., Regelous, M., 2011. Confirmation of massindependent $\mathrm{Ni}$ isotopic variability in iron meteorites. Geochimica et Cosmochimica Acta 75 (24), 7906-7925.

Thiemens, M. H., 1999. Mass-independent isotope effects in planetary atmospheres and the early solar system. Science 283 (5400), $341-345$.

Travaglio, C., Hillebrandt, W., Reinecke, M., Thielemann, F., 2004. Nucleosynthesis in multi-dimensional SN Ia explosions. Astronomy and Astrophysics 425, 1029-1040.

Travaglio, C., Röpke, F. K., Gallino, R., Hillebrandt, W., 2011. Type ia supernovae as sites of the p-process: Two-dimensional models coupled to nucleosynthesis. The Astrophysical Journal 739 (2), 93.

Trinquier, A., Birck, J. L., Allègre, C. J., Göpel, C., Ulfbeck, D., 2008. ${ }^{53} \mathrm{Mn}-{ }^{53} \mathrm{Cr}$ 
systematics of the early Solar System revisited. Geochimica et Cosmochimica Acta 72 (20), 5146-5163.

Trinquier, A., Elliott, T., Ulfbeck, D., Coath, C., Krot, A. N., Bizzarro, M., 2009. Origin of nucleosynthetic isotope heterogeneity in the Solar protoplanetary disk. Science 324 (5925), 374-376.

Umeda, H., Nomoto, K., 2002. Nucleosynthesis of zinc and iron peak elements in population III type II supernovae: Comparison with abundances of very metal poor halo stars. The Astrophysical Journal 565, 385404.

Usui, T., Iwamori, H., 2013. Mixing relations of the howardite-eucrite-diogenite suite: A new statistical approach of independent component analysis for the dawn mission. Meteoritics 85 Planetary Science 48 (11), 2289-2299.

Völkening, J., Papanastassiou, D. A., 1989. Iron isotope anomalies. The Astrophysical Journal, Letters 347, L43-L46.

Wasson, J. T., Kallemeyn, G. W., 1988. Compositions of chondrites. Philosophyical Transactions of the Royal Society AA325, $535-544$.

Williams, N., Schönbächler, M., Fehr, M., Akram, A., Parkinson, I., et al., 2014. Different heterogeneously distributed titanium isotope components in solar system materials and mass-dependent titanium isotope variations. In: Lunar and Planetary Institute Science Conference Abstracts. Vol. 45. p. 2183 .

Woosley, S. E., 1997. Neutron-rich Nucleosynthesis in Carbon Deflagration Supernovae. The Astrophysical Journal 476 (2), 801810.

Wurm, G., Teiser, J., Bischoff, A., Haack, H., Roszjar, J., 2010. Experiments on the photophoretic motion of chondrules and dust 
Appendix: Table of Renormalized Hibonite Ti Isotope Compositions

\begin{tabular}{|c|c|c|c|c|c|c|c|c|}
\hline Sample Name & Type & Reference & $\varepsilon^{46} \mathrm{Ti} \frac{47}{49}$ & 2 s.e. & $\varepsilon^{48} \mathrm{Ti} \frac{47}{49}$ & 2 s.e. & $\varepsilon^{50} \mathrm{Ti} \frac{47}{49}$ & 2 s.e. \\
\hline $7-76$ & SHIB & Ireland 1990 & -6.1 & 64.6 & 2.0 & 29.8 & 36.9 & 87.1 \\
\hline $7-143$ & SHIB & Ireland 1990 & 8.1 & 8.7 & 2.0 & 4.7 & 28.1 & 14.5 \\
\hline $7-290$ & SHIB & Ireland 1990 & 39.6 & 68.8 & 6.9 & 31.1 & -57.4 & 91.5 \\
\hline $7-373$ & SHIB & Ireland 1990 & -9.8 & 64.9 & 7.6 & 27.7 & -4.8 & 79.2 \\
\hline $7412 \mathrm{t}$ & PLAC & Ireland 1990 & 5.4 & 7.1 & 14.6 & 4.1 & 40.4 & 14.0 \\
\hline $7-505$ & SHIB & Ireland 1990 & -4.3 & 44.3 & 12.1 & 19.6 & 25.8 & 57.8 \\
\hline $7-551$ & SHIB & Ireland 1990 & -1.1 & 59.7 & 3.0 & 26.9 & 33.0 & 83.7 \\
\hline $7-644$ & PLAC & Ireland 1990 & 69.5 & 80.5 & 31.5 & 33.8 & -13.0 & 93.3 \\
\hline $7-658$ & PLAC & Ireland 1990 & 58.0 & 63.9 & 46.7 & 28.7 & 55.9 & 84.4 \\
\hline $7-664$ & SHIB & Ireland 1990 & 6.3 & 8.9 & 21.6 & 5.2 & 22.3 & 17.5 \\
\hline $7-734$ & SHIB & Ireland 1990 & 11.9 & 49.7 & 20.1 & 22.0 & 25.9 & 63.4 \\
\hline $7-789$ & SHIB & Ireland 1990 & 5.8 & 5.5 & 16.1 & 3.3 & 11.9 & 12.7 \\
\hline $7-821$ & SHIB & Ireland 1990 & 20.9 & 51.4 & -0.6 & 23.3 & 58.7 & 69.1 \\
\hline $7-953$ & SHIB & Ireland 1990 & 9.4 & 9.0 & 16.6 & 5.2 & 16.4 & 18.0 \\
\hline $7-980$ & PLAC & Ireland 1990 & -10.1 & 10.7 & 23.7 & 6.4 & -110.0 & 18.1 \\
\hline $7-981$ & PLAC & Ireland 1990 & -16.1 & 7.1 & 19.7 & 4.1 & -112.0 & 12.8 \\
\hline 7-A84 & SHIB & Ireland 1990 & -8.1 & 38.9 & -2.0 & 18.0 & 24.9 & 52.9 \\
\hline 7-A95 & SHIB & Ireland 1990 & 30.2 & 59.9 & -9.6 & 24.8 & -53.7 & 69.6 \\
\hline $8-47$ & SHIB & Ireland 1990 & 12.6 & 40.8 & 7.5 & 18.7 & 7.6 & 53.4 \\
\hline 849 & SHIB & Ireland 1990 & 50.9 & 64.2 & 7.9 & 29.0 & -34.3 & 77.8 \\
\hline $8-65$ & SHIB & Ireland 1990 & 5.1 & 44.7 & -1.0 & 19.5 & 19.1 & 59.0 \\
\hline $8-66$ & PLAC & Ireland 1990 & -24.3 & 66.6 & 23.8 & 27.9 & -208.0 & 80.3 \\
\hline $13-02$ & SHIB & Ireland 1990 & -11.0 & 45.0 & -9.0 & 20.6 & 28.9 & 69.1 \\
\hline $13-03$ & SHIB & Ireland 1990 & 4.2 & 29.4 & -6.0 & 14.2 & 20.1 & 43.3 \\
\hline $13-04$ & SHIB & Ireland 1990 & -16.5 & 31.2 & 12.1 & 14.9 & 29.6 & 45.0 \\
\hline $13-13$ & PLAC & Ireland 1990 & 119.0 & 59.7 & -38.2 & 24.0 & 2491.0 & 68.9 \\
\hline $13-23$ & BAG & Ireland 1990 & -40.2 & 45.8 & 5.7 & 19.1 & -178.0 & 50.6 \\
\hline $13-24$ & SHIB & Ireland 1990 & -33.0 & 69.0 & 2.7 & 31.4 & 146.0 & 93.5 \\
\hline $13-25$ & PLAC & Ireland 1990 & 64.8 & 64.6 & 40.1 & 29.3 & -350.0 & 85.9 \\
\hline $13-33$ & SHIB & Ireland 1990 & -69.5 & 55.5 & -40.2 & 25.8 & 0.0 & 80.6 \\
\hline $13-37$ & SHIB & Ireland 1990 & -10.5 & 43.4 & -9.5 & 19.5 & 13.5 & 54.0 \\
\hline $13-51$ & PLAC & Ireland 1990 & 40.5 & 82.6 & 47.8 & 36.5 & -379.0 & 104.0 \\
\hline $13-60$ & SHIB & Ireland 1990 & -6.6 & 37.2 & -3.5 & 16.7 & -24.5 & 46.3 \\
\hline $13-61$ & SHIB & Ireland 1990 & 23.7 & 39.6 & -17.1 & 17.3 & -5.4 & 48.5 \\
\hline 1412 & PLAC & Ireland 1990 & -46.1 & 48.9 & -0.3 & 22.3 & -210.0 & 63.5 \\
\hline $14-14$ & SHIB & Ireland 1990 & -4.1 & 33.6 & 2.0 & 14.8 & 44.9 & 43.1 \\
\hline Mur-A1 & - & Zinner 1986 & -61.9 & 27.3 & -18.3 & 11.6 & 70.9 & 32.1 \\
\hline Mur-H7 & - & Zinner 1986 & 15.5 & 12.6 & -15.1 & 9.1 & 58.2 & 31.4 \\
\hline Mur-H8 & - & Zinner 1986 & -7.9 & 41.0 & -14.0 & 16.8 & -1.9 & 48.7 \\
\hline Mur-20 & - & Zinner 1986 & 4.7 & 7.0 & 23.1 & 4.1 & -433.0 & 13.9 \\
\hline Mur-70 & - & Zinner 1986 & -10.7 & 6.5 & 30.3 & 4.8 & -222.0 & 15.6 \\
\hline Mur-170 & - & Zinner 1986 & 33.2 & 6.8 & -6.6 & 3.6 & -539.0 & 12.0 \\
\hline My-H3 & - & Zinner 1986 & 48.1 & 25.8 & -11.2 & 11.7 & 955.0 & 35.5 \\
\hline My-H4 & - & Zinner 1986 & 47.0 & 58.5 & -10.2 & 23.6 & 971.0 & 65.0 \\
\hline CH-B2 & PLAC & Sahijpal 2000 & -4.8 & 39.4 & -17.0 & 18.4 & 502.0 & 65.1 \\
\hline CH-B7 & PLAC & Sahijpal 2000 & 99.8 & 43.2 & -11.8 & 21.4 & 121.0 & 79.9 \\
\hline CH-A5 & SHIB & Sahijpal 2000 & 11.1 & 38.3 & -28.6 & 16.3 & -15.8 & 47.9 \\
\hline CH-B5 & PLAC & Sahijpal 2000 & 21.7 & 37.6 & -25.1 & 22.3 & 138.0 & 65.1 \\
\hline All-3529-42 & - & Sahijpal 2000 & 13.6 & 53.4 & 10.5 & 25.1 & 22.6 & 72.1 \\
\hline CH-A3 & SHIB & Sahijpal 2000 & -11.3 & 73.8 & -22.5 & 35.9 & 86.4 & 109.0 \\
\hline CH-B3 & PLAC & Sahijpal 2000 & 7.8 & 46.5 & -11.6 & 22.0 & 92.5 & 75.7 \\
\hline CH-A4 & SHIB & Sahijpal 2000 & 2.1 & 71.2 & -35.5 & 34.9 & 577.0 & 115.0 \\
\hline Mur-S15 & SHIB & Liu 2009 & -96.9 & 71.9 & -27.1 & 35.2 & 15.3 & 110.0 \\
\hline Mur-P1 & PLAC & Liu 2009 & 23.5 & 78.8 & -3.1 & 41.8 & 496.0 & 133.0 \\
\hline Mur-P2 & PLAC & Liu 2009 & -50.1 & 85.7 & -29.8 & 41.8 & 232.0 & 133.0 \\
\hline Mur-P6 & PLAC & Liu 2009 & -113.0 & 79.0 & 38.1 & 37.4 & -39.8 & 117.0 \\
\hline Mur-P7 & PLAC & Liu 2009 & -140.0 & 75.4 & -35.8 & 38.0 & 2058.0 & 116.0 \\
\hline Mur-P8 & PLAC & Liu 2009 & -181.0 & 79.4 & 5.2 & 38.2 & 38.6 & 119.0 \\
\hline Mur-P9-spot1 & PLAC & Liu 2009 & -273.0 & 94.8 & 30.5 & 44.4 & -335.0 & 130.0 \\
\hline Mur-P9-spot2 & PLAC & Liu 2009 & -245.0 & 74.4 & 53.1 & 35.9 & -410.0 & 107.0 \\
\hline Mur-B1-spot1a & BAG & Liu 2009 & 123.0 & 105.0 & 37.4 & 47.6 & -50.2 & 139.0 \\
\hline
\end{tabular}




\begin{tabular}{|c|c|c|c|c|c|c|c|c|}
\hline Mur-B1-spot1-1a & BAG & Liu 2009 & 138.0 & 117.0 & 44.9 & 51.4 & -143.0 & 140.0 \\
\hline Mur-B1-spot2a & BAG & Liu 2009 & 54.1 & 108.0 & 34.5 & 53.3 & -449.0 & 159.0 \\
\hline Mur-B1-spot2-1a & BAG & Liu 2009 & -72.6 & 122.0 & 9.9 & 54.4 & -247.0 & 145.0 \\
\hline Mur-B1-spot3 & $\mathrm{BAG}$ & Liu 2009 & -35.3 & 85.9 & -10.9 & 39.9 & -113.0 & 110.0 \\
\hline Mur-B1-spot3-1 & BAG & Liu 2009 & -0.9 & 94.1 & 48.5 & 44.4 & -16.0 & 126.0 \\
\hline MUR-AL-a & - & Fahey 1985 & 7.1 & 65.9 & 1.0 & 26.2 & 82.1 & 73.7 \\
\hline MUR-Al-B & - & Fahey 1985 & -9.6 & 29.0 & -0.5 & 12.8 & 48.4 & 35.7 \\
\hline MUR-H7 & - & Fahey 1985 & 15.5 & 25.2 & -15.1 & 10.3 & 58.2 & 27.7 \\
\hline MY-H3-a & - & Fahey 1985 & 32.7 & 29.0 & -10.1 & 13.0 & 971.0 & 41.5 \\
\hline MY-H3-b & - & Fahey 1985 & 103.0 & 56.3 & -12.8 & 24.7 & 902.0 & 68.1 \\
\hline ATP-1 & - & Fahey 1985 & -25.4 & 23.8 & 1.1 & 10.2 & 10.6 & 29.8 \\
\hline ATP-2-a & - & Fahey 1985 & -27.5 & 36.9 & 3.1 & 16.3 & -1.5 & 52.1 \\
\hline ATP-2-b & - & Fahey 1985 & -0.3 & 46.9 & 16.1 & 18.3 & 17.8 & 49.3 \\
\hline ATP-3-a & - & Fahey 1985 & 15.4 & 35.1 & -7.1 & 15.5 & 21.3 & 44.6 \\
\hline ATP-3-b & - & Fahey 1985 & 35.5 & 32.1 & 8.9 & 14.6 & 25.3 & 42.5 \\
\hline $\mathrm{C}-1$ & FUN & Niederer 1980 & -10.6 & 1.6 & 0.6 & 0.7 & -39.0 & 1.8 \\
\hline $\mathrm{C}-2$ & FUN & Niederer 1980 & -11.8 & 1.8 & 1.2 & 0.8 & -37.9 & 2.8 \\
\hline EK-1-4-1 & FUN & Niederer 1980 & -13.2 & 4.4 & -15.5 & 2.5 & 17.6 & 9.7 \\
\hline EK-1-4-2 & FUN & Niederer 1980 & -9.8 & 3.4 & -14.3 & 1.7 & 16.1 & 4.8 \\
\hline EK-1-4-3 & FUN & Niederer 1980 & -7.7 & 11.3 & -18.0 & 6.4 & 15.1 & 19.3 \\
\hline EK-1-4-4 & FUN & Niederer 1980 & -6.1 & 5.6 & -16.4 & 2.6 & 10.0 & 7.3 \\
\hline EK-1-4-5 & FUN & Niederer 1980 & -8.8 & 4.8 & -16.3 & 2.1 & 14.8 & 5.4 \\
\hline DJ-1 & - & Hinton 1987 & -2.7 & 22.3 & 6.5 & 15.2 & -190.0 & 58.9 \\
\hline BB-5 & - & Hinton 1987 & -7.1 & 23.8 & 52.6 & 16.3 & -578.0 & 56.9 \\
\hline BB-5-c & - & Hinton 1987 & -0.6 & 26.7 & 58.1 & 10.3 & -573.0 & 25.6 \\
\hline Gr-1-r & - & Hinton 1987 & 39.1 & 23.5 & 7.4 & 12.3 & -93.8 & 53.8 \\
\hline Gr-1-c & - & Hinton 1987 & 34.3 & 37.9 & -9.7 & 16.0 & -105.0 & 44.7 \\
\hline $\mathrm{SH}-7$ & - & Hinton 1987 & 1.6 & 20.3 & -3.5 & 7.7 & 160.0 & 17.3 \\
\hline Mur-H9 & - & Fahey 1987a & -26.8 & 32.0 & -7.4 & 14.1 & 147.0 & 42.8 \\
\hline My-CH1 & - & Fahey 1987a & -2.0 & 23.4 & -4.0 & 10.2 & 30.0 & 29.5 \\
\hline MY-IP & - & Fahey 1987a & 5.0 & 47.3 & 3.0 & 19.8 & -1.0 & 56.0 \\
\hline CB-H2 & - & Fahey 1987a & -1.1 & 24.2 & 5.0 & 10.9 & -3.1 & 36.1 \\
\hline CB-H4 & - & Fahey 1987a & 17.4 & 35.3 & -5.1 & 16.0 & 133.0 & 48.2 \\
\hline HAL-Ha & - & Fahey 1987a & 13.4 & 71.0 & 18.6 & 30.4 & 188.0 & 87.0 \\
\hline HAL-Hb & - & Fahey 1987a & 30.0 & 52.1 & 6.4 & 23.9 & 124.0 & 73.3 \\
\hline DA2-12 & - & Fahey 1987a & 32.3 & 35.0 & -15.6 & 15.5 & 49.9 & 45.0 \\
\hline ATP-1b & - & Fahey 1987a & 5.5 & 20.5 & 4.5 & 8.5 & 23.5 & 23.8 \\
\hline MUR-H! & Hib & Fahey $1987 \mathrm{~b}$ & 17.7 & 23.7 & -29.1 & 10.3 & 65.2 & 30.3 \\
\hline BB-5-H & Hib & Fahey 1987b & -34.7 & 27.6 & 59.9 & 12.8 & -574.0 & 37.4 \\
\hline BB-5-C & Corr & Fahey 1987b & -20.8 & 46.8 & 50.1 & 20.7 & -581.0 & 58.4 \\
\hline
\end{tabular}

Table 2:: Table showing Ti isotope compositions of hibonites renormalized from literature. Errors were obtained using a Monte Carlo simulation as outlined in the text. 